\title{
Corela
}

Cognition, représentation, langage

HS-32 | 2020

Les postures énonciatives

\section{Postures énonciatives et rôles interactionnels des modérateurs dans les débats télévisés politiques}

\section{Ida Hekmat}

\section{(2) OpenEdition}

\section{Journals}

Édition électronique

URL : https://journals.openedition.org/corela/12382

DOI : 10.4000/corela.12382

ISSN : 1638-573X

Éditeur

Cercle linguistique du Centre et de l'Ouest - CerLICO

\section{Référence électronique}

Ida Hekmat, «Postures énonciatives et rôles interactionnels des modérateurs dans les débats télévisés politiques », Corela [En ligne], HS-32 | 2020, mis en ligne le 09 novembre 2020, consulté le 14 juillet 2021. URL : http://journals.openedition.org/corela/12382 ; DOI : https://doi.org/10.4000/corela. 12382

Ce document a été généré automatiquement le 14 juillet 2021.

\section{c) (i) (2)}

Corela - cognition, représentation, langage est mis à disposition selon les termes de la licence Creative Commons Attribution - Pas d'Utilisation Commerciale - Partage dans les Mêmes Conditions 4.0 International. 


\title{
Postures énonciatives et rôles interactionnels des modérateurs dans les débats télévisés politiques
}

\author{
Ida Hekmat
}

1 Les fonctions des modérateurs et modératrices de débats télévisés au sein des interactions polémiques qu'ils animent sont diverses et complexes. Leurs rôles interactionnels ont été décrits dans la littérature (Doury 1995, Pérennec 2001, Clayman et Heritage 2002, Kerbrat-Orecchioni 2005, 2017), mettant en évidence, comme le dit Pérennec, qu'ils sont " plus qu'une simple courroie de transmission », " avant tout [des] acteur[s] du monde médiatique » (Pérennec 2001 : 21) :

a) Ils font partie de la chaîne d'émetteurs responsables de l'émission et participent notamment, en amont du débat, à la sélection des débattants qui façonne remarquablement l'affrontement des points de vue durant le débat. Ils font office d'hôtes et assument les séquences d'ouverture et de clôture, face caméra, pendant le débat.

b) Ils sont les maîtres (parfois contestés) du jeu durant le débat. En effet, ils proposent, posent, imposent parfois, divers éléments de l'interaction : distribution de la parole, objets de discours, identités discursives des invités etc., étant entendu que ces aspects peuvent être négociés par les interactants (Kerbrat-Orecchioni 2005). Ils sont également metteurs en scène de la polémique, cherchant à favoriser la « guerre verbale» (Kerbrat-Orecchioni 1980) ${ }^{1}$.

c) Enfin, ils sont les médiateurs entre les débattants et les téléspectateurs (qui sont les récepteurs visés mais absents), cherchant à clarifier les propos tenus et les positions défendues.

2 Ainsi, du point de vue du volume de parole dont ils disposent, ils sont des sous-locuteurs, aux plans interactionnel et argumentatif, ils sont en position haute, participant fortement à la structuration, aussi bien interactionnelle que thématique, des échanges, alors qu'au plan énonciatif, leur discours est marqué (comme la plupart des discours journalistiques) par des stratégies d'«effacement énonciatif» (Vion 2001, Rabatel 2004b) qui contribuent à donner l'impression qu'ils sont hors débat. 
3 Dans le sillage des travaux s'intéressant à l'effacement énonciatif, qui montrent que cet effacement relève d'une stratégie de gommage des traces de la subjectivité du sujet dans son discours et non d'une absence de subjectivité (nous renvoyons notamment à Langages 156 (Rabatel 2004a) et Semen 17 (Amossy et Koren 2004a)), nous nous penchons sur l'apparente contradiction marquant le rôle discursif des modérateurs : ils semblent être hors débat alors qu'ils structurent les échanges. Nous nous intéressons dans cet article $^{2}$, aux segments où les modérateurs représentent ${ }^{3}$ les discours tenus par les débattants in praesentia, ces occurrences constituant les formes de dialogisme majoritaires dans leurs discours: les modérateurs semblent distribuer la parole, la reformuler, permettre sa circulation ainsi que la confrontation des points de vue en étant tout à fait neutres par rapport aux échanges. Comme l'ont montré les travaux cités, cette neutralité n'est qu'apparente : pour étudier en détail cette question et les fonctionnements énonciatifs, interactionnels et argumentatifs précis à l'œuvre, nous analysons les segments évoqués à partir des postures énonciatives, telles qu'elles ont été définies par Rabatel (voir entre autres Rabatel 2004b, 2017).

4 La question énonciative abordée, qui s'ancre dans la problématique plus générale de la responsabilité discursive et éthique (Amossy et Koren 2004b, Koren 2004, Rabatel et Chauvin-Vileno 2006, Rabatel et Monte 2017, Rabatel 2017, Yanoshevsky 2018, Koren 2019) n'est pas dénuée de conséquences aux plans politique et social. En effet, les débats télévisés qui nous occupent sont des débats politiques au sens où il traitent de questions ayant trait à la polis - ils ne constituent donc pas des débats culturels ou des débats de divertissement ${ }^{4}$-, bien que les débattants qui y participent ne soient pas uniquement des hommes et des femmes politiques et bien que ces débats ne relèvent pas du parangon du genre que représentent les débats télévisés de l'entre-deux-tours de l'élection présidentielle (pour une analyse de ce genre discursif précis, voir KerbratOrecchioni 2017). En outre, ces débats télévisés jouent un rôle particulier quant à la conflictualité sociale : ils se proposent, en différé (s'opposant ainsi à l'immédiateté qui caractérise d'autres genres de discours médiatiques), d'offrir aux téléspectateurs un panorama des différents points de vue concernant une question débattue dans l'espace public. Toutefois, leur orientation vers la polémique (Kerbrat-Orecchioni 1980, Amossy et Burger 2011) n'est pas uniquement au service d'une logique d'information, elle sert aussi une logique de captation, dont la dérive possible vers le sensationnalisme est régulièrement critiquée (voir par exemple Clayman et Heritage 2002 : 335).

5 Le corpus d'étude est constitué de 4 débats télévisés, français et allemands, diffusés en 2006 et traitant de ce qui fut appelé « l'affaire des caricatures de Mahomet $»^{5}$ :

- Hart aber fair, « Die Moslem-Angst, kommt der Krieg der Kulturen?»/« La peur du/ des musulman(s) : la guerre des civilisations approche-t-elle?», WDR, 08.02.2006

- Menschen bei Maischberger, «Gesinnungstest für Deutschland, brauchen wir mehr Intoleranz?»/«test de mentalité pour l'Allemagne: avons-nous besoin de plus d'intolérance ? », ARD, 07.02.2006

- Mots Croisés, « L'Islam, les raisons de la colère », France 2, 06.02.2006

- Pièces à conviction, "Caricatures: les dessous d'un embrasement », France 3, 31.03.2006

6 Notre étude met en avant la mobilité posturale à l'œuvre dans la représentation par les modérateurs des discours tenus par les débattants in praesentia et confirme l'impossibilité de lier de façon univoque une certaine posture énonciative à un rôle ou un genre discursifs (Rabatel 2015) : les modérateurs représentent les discours des invités, dans un geste de synthèse, pour les clarifier autant que pour favoriser la 
polémique, en préservant leur éthos ${ }^{6}$ de neutralité ${ }^{7}$, matérialisé en discours par l'effacement énonciatif qui exclut a priori les formes explicites d'accord ou de prise en charge. Nous aborderons dans un premier temps les cas, majoritaires, où les modérateurs sur-énoncent afin de favoriser la polémique, pour observer dans un deuxième temps un passage où la sur-énonciation masque l'accord. Enfin, nous nous pencherons sur les cas de sous-énonciation, qui sont pragmatiquement toujours liés à des demandes de validation.

\section{Sur-énonciation et polémisation de l'interaction}

7 Les cas de sur-énonciation sont majoritaires : le locuteur/énonciateur 1 (L1/E1) ${ }^{8}$ (ici le modérateur) représente le point de vue d'un autre ou d'autres - ici d'un ou de débattant(s) - en "paraissant dire la même chose tout en modifiant à son profit le domaine de pertinence ou [l']orientation argumentative" (Rabatel 2015: 127, nous soulignons). Ces reformulations peuvent être des représentations par les modérateurs $\mathrm{du}$ discours déjà tenu par $\mathrm{X}$ à $\mathrm{X}$ lui-même (extraits 1 et 2 ) ou une représentation du discours de X à Y (extrait 1). Dans les deux cas, la position de surplomb tient à la polémisation des dires de l'autre : le «profit » que le modérateur tire de la façon dont il représente les discours des débattants réside moins dans le fait qu'il peut soutenir son point de vue propre que dans le fait que, choisissant de représenter telle partie du discours de l'autre ou telle autre, choisissant un certain type de reformulation, choisissant aussi d'adresser le discours au débattant $\mathrm{X}$ ou $\mathrm{Y}$, il favorise la polémique. Cette polémisation est constitutive de son rôle argumentatif au sein des débats, et permet donc la construction d'un éthos de compétence, mais peut aussi, si la reformulation des discours tenus s'avère trop polémique, le mettre en danger, comme on le verra grâce à la stratégie de réparation avec l'extrait 2 .

8 Le premier extrait présente le cas le plus fréquent observé : la sur-énonciation à l'œuvre permet de radicaliser les discours représentés, c'est-à-dire de les rendre moins nuancés, de façon à favoriser l'affrontement entre les interactants.

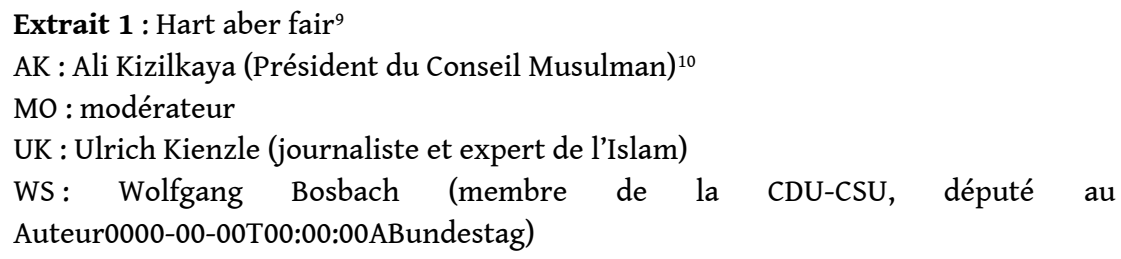

9 Le topic général est ici celui des intentions (cachées) des acteurs de « l'affaire » : avant cet extrait, Wolfang Bosbach met en cause les manifestations de protestation contre les « caricatures » en expliquant qu'elles sont mises en scène par les régimes en place dans les pays arabes. Alors qu'Ulrich Kienzle, ici en (1), développe le topic en proposant un topic articulé au précédent (les intentions du journal Jyllands Posten), le modérateur remet au centre du débat la question de la mise en scène des manifestations à des fins politiques en (3). On note les relances du topic en (5) et (7). En (9) il ouvre une nouvelle partie de la séquence ${ }^{11}$ en donnant la parole à Ali Kizilkaya. Au début du tour, on trouve une qualification des dires antérieurs de Kienzle : selon le modérateur, l'invité exprime "un reproche de taille", sans que soit nommée la cible du reproche. Si reproche il y a dans les tours précédents de Kienzle, il s'adresse aux régimes qui selon lui mettent en scène les manifestations. Mais la non-mention du destinataire permet au modérateur d'actualiser l'acte menaçant sur la scène interactionnelle immédiate, puisqu'il semble 
viser Ali Kizilkaya. Pour ce faire, le modérateur introduit deux discours autres et adopte une posture de sur-énonciation, modifiant les discours ou les sources auxquelles ils sont attribués afin de durcir les positions des uns et des autres et favoriser l'affrontement entre débattants (comme le prouve le tour (11) où le modérateur invite Kizilkaya à se prononcer explicitement sur la justesse ou non des positions de Bosbach) :

- En (9), après l'adresse à Ali Kizilkaya, le modérateur représente les dires que celuici a tenus précédemment ("vous nous avez expliqué au moment où nous avons décrit les dessins pourquoi vous vous sentiez (..) vraiment touché personnellement »). Or le discours attribué à Kizilkaya efface les subtilités de l'interaction telle qu'elle a eu lieu : c'est le modérateur qui avait proposé à l'invité un éthos émotionné et avait fini, après que Kizilkaya avait tenté de négocier son éthos, par le lui imposer ${ }^{12}$. Par la sur-énonciation visible ici, le modérateur peut inclure Kizilkaya au groupe dont l'indignation est présentée comme insincère.

- Durcissant la position représentée de Kizilkaya, il durcit également la position de ceux qui le mettraient en cause : le deuxième discours représenté est attribué à un locuteur indéfini et pluriel («viele»/«de nombreuses personnes»), qui est explicitement mis en relation avec la situation d'énonciation présente (" nous entendons maintenant ») et renvoie ainsi aux deux locuteurs in praesentia qui ont endossé cette position (voir notamment (3) et (4)) : Wolfgang Bosbach et Ulrich Kienzle. Le pronom « viele » amplifie donc le locuteur à qui est attribué le point de vue, qui, partant, est lui aussi amplifié : d'un côté, on peut considérer que la face des deux invités en question est préservée (puisque ce ne sont pas eux qui sont représentés comme mettant en cause explicitement Kizilakaya) et d'un autre côté, leur thèse, présentée comme consensuelle, apparait ici comme particulièrement pertinente. La caractérisation de l'indignation que le modérateur impute au locuteur indéfini «viele» est en outre marquée par une structure redoublée qui vise à conférer une certaine efficacité rhétorique au discours représenté: «l'indignation est organisée instrumentalisée ».

10 On trouve dans l'extrait suivant un autre cas de sur-énonciation, où le modérateur adresse à un débattant une reformulation des dires qu'il vient de tenir tout en gommant les nuances de son propos. L'orientation polémique du discours représenté ${ }^{13}$ peut nuire à l'éthos du débattant, mais aussi à celui du modérateur, qui, en même temps qu'il sur-énonce - en le radicalisant - le discours reformulant, prend soin de (re)construire son éthos de vigilance et de probité.

Extrait $2:$ Mots croisés

$\mathrm{CD}$ : Christophe Deloire (journaliste au Point, auteur de Les islamistes sont déjà là.

Enquête sur une guerre secrète)

MO : Auteur0000-00-00T00:00:00Amodérateur

11 En (2), Christophe Deloire répond à la question posée par le modérateur en (1). Ce dernier soumet un point de vue à l'invité, sans qu'il soit possible de dire s'il le prend en charge lui-même ou pas. Néanmoins, le fait même que la question soit posée par le modérateur la constitue comme une question pertinente dans le contexte. L'invité valide le point de vue proposé mais avec mesure : d'une part, il indique que «c'est un peu tôt pour le dire» et d'autre part, il emploie la locution figée «faire feu de tout bois» par laquelle il prend implicitement ses distances avec la proposition du modérateur: il ne s'agit plus dans son expression de la finalité de "l'affaire des caricatures ", mais de son mésusage possible. Ce palier d'élan argumentatif est ensuite illustré par un ensemble d'exemples et aboutit à une conclusion prudente de l'invité : «c'est par euh par de tels biais (.) qu'un ordre social (...) peut être (.) progressivement imposé ", après quoi Christophe Deloire entreprend de changer de topic. En (3), le 
modérateur prend la parole en chevauchement et présente une reformulation synthétisante (comme l'indique la locution "au bout du compte») du discours précédent. Alors que la formulation de Deloire constitue une tournure générale, sa représentation par le modérateur se fait sous forme d'un groupe nominal, qui est répété en fin de tour et actualisé, rattaché à la situation du nous, par le complément du nom « de la société française ». On remarque, dans le discours représenté, l'effacement (i) de l'adverbe "progressivement", qui suggérait dans le discours de Deloire une possibilité de contrôle sur la situation, et (ii) de l'expression de la virtualité exprimée par le verbe de modalité " pouvoir ». La sur-énonciation tient ici à la modification par le modérateur du point de vue représenté en accord avec son propre but: rendre le point de vue de Deloire davantage susceptible de susciter le désaccord, c'est-à-dire le rendre polémique. Si le geste de synthèse est conforme au rôle interactionnel du modérateur et qu'il participe d'un éthos positif, la radicalisation opérée peut nuire à l'image de modérateur respectueux de la parole des invités. L'incise, présente entre les segments représentant le discours autre, semble devoir résoudre cette contradiction. Elle ne participe pas de la sur-énonciation mais relève d'une stratégie de restauration de l'éthos mis à mal par la sur-énonciation: «il faut employer des termes (...) extrêmement réfléchis et en faisant attention à ce qu'on dit ». Dans ce commentaire métadiscursif, l'emploi du pronom "on " constitue en injonction générale ce qui semble dans un premier temps être adressé à Deloire: l'incise interrompt une représentation en sur-énonciation du discours de Deloire - par laquelle le modérateur polémise fortement ses dires - et enjoint à la responsabilité discursive. Dans le dire comme dans le dit, le modérateur adopte une position de surplomb et se pose comme gardien des normes éthiques, tout en ayant lui-même, en sur-énonçant, attribué à l'invité un discours les enfreignant.

La sur-énonciation consiste ici en la représentation sélective et orientée des propos tenus par les débattants: il s'agit de clarifier les positions en les rendant plus prégnantes et, par là, de favoriser la polémique en rendant les positions davantage susceptibles d'être contestées. Eu égard au genre discursif et au rôle interactionnel des modérateurs, ces cas de sur-énonciation sont attendus, autant que la posture de sousénonciation, abordée dans le troisième temps de notre étude. Ils ont pour point commun de ne pas comporter de marques d'accord de la part du locuteur/énonciateur premier. Nous abordons à présent un passage témoignant d'un phénomène plus rare mais significatif : le déroulement de la séquence témoigne d'un accord du locuteur/ énonciateur premier avec les dires représentés en sur-énonciation.

\section{De la sur-énonciation polémisante à l'accord}

Au premier abord, on observe dans le passage suivant un fonctionnement similaire à ceux observés pour les extraits 1 et 2 : le modérateur sur-énonce, afin de polémiser l'échange, les dires attribués à deux invités en les adressant à une débattante. Pourtant, si on prend en compte, plus globalement, la séquence, il faut interpréter la surénonciation comme masquant un accord du locuteur/énonciateur premier avec les dires qu'il met en scène. Malgré l'accord, interprété a posteriori au vu du déroulement de la séquence, il nous semble plus juste de parler de sur-énonciation masquant l'accord que de co-énonciation ou de sur-énonciation recouvrant la co-énonciation ${ }^{14}$ : l'accord impliqué ici est reconstruit par l'interprétant dans l'après-coup, et il n'est pas 
insignifiant que ce soit une stratégie de sur-énonciation suivie par une marque subtile d'accord qui soit retenue par le modérateur. D'une part parce que le phénomène de surénonciation est majoritaire dans le corpus, d'autre part parce que, comme le dit Florea, et notre analyse le confirme, la co-énonciation s'accommode mal de l'effacement énonciatif visant à préserver l'éthos de neutralité des modérateurs (Florea 2015 : note p. 352).

On trouve dans cet extrait, du point de vue du topic abordé, ce qui est devenu un topos dans les discours médiatiques sur l'Islam depuis plusieurs années: l'appel aux musulmans à se désolidariser explicitement des auteurs de violences. Ce topos est prégnant dans notre corpus mais aussi circulant au sein des moments discursifs suivants : on se rappelle la campagne «not in my name " sur les réseaux sociaux en 2014, qui semblait répondre à cette injonction répétée, mais aussi les discours qui, après les meurtres de Charlie Hebdo, considéraient que ne pas affirmer qu'on "est Charlie » revenait à prendre position pour le terrorisme se réclamant de l'islam ${ }^{15}$.

Extrait 3 : Mots croisés

PL : Pierre Lellouche (député UMP de Paris, président de l'Assemblée Parlementaire de l'OTAN)

PT : Philippe Tesson (présenté par le modérateur comme «notre confrère journaliste et éditorialiste »)

SK: Saida Kada (membre du collectif «Diversité", dirige une entreprise informatique, auteure de L'une voilée l'autre Auteur0000-00-00T00:00:00Apas)

Pierre Lellouche et Philippe Tesson se sont à plusieurs reprises opposés à Saïda Kada en amont de ce passage. Alors que Tesson lui répond en (2) que la majorité des musulmans est trop silencieuse, Kada s'oppose à lui en se posant comme représentante de cette majorité en (3) - qui n'est, partant, pas silencieuse - et développe le thème du dialogue avec les musulmans, avant d'en appeler à un usage responsable des mots. En (10) le modérateur reprend ce qu'il a initié en (8) : il représente le discours de Pierre Lellouche et Philippe Tesson qu'il adresse directement à Saïda Kada. Le topic du silence de la supposée communauté musulmane est bien évoqué par Philippe Tesson en (2), même si on ne trouve rien d'explicite dans le contexte amont concernant Pierre Lellouche. En (10), le modérateur attribue aux deux invités une injonction, atténuée par l'usage du verbe "suggérer»: ce qui est posé ici, c'est que ce qui est considéré comme la " communauté musulmane » ne rompt pas ou pas assez clairement avec les terroristes, ce qui implique qu'elle est solidaire, voire co-responsable des actes que ceux-ci commettent. Du point de vue local, on voit ici un phénomène de sur-énonciation similaire à ce que nous avons observé dans la première partie de cette étude: le modérateur semble reprendre les dires des invités qu'il nomme mais les réoriente dans un but de polémisation, en formulant une injonction qu'il adresse directement à une débattante. Mais en prenant en compte le cotexte, on note que cette apparente surénonciation relève d'une stratégie masquant l'accord du modérateur avec les débattants, dont il met en scène le discours, et de désaccord avec Saïda Kada :

- en amont: il exprime explicitement en (4), malgré l'absence de marques personnelles qui gomment sa prise de position, son désaccord avec Kada ;

- en aval : si le modérateur semble continuer le discours attribué à Pierre Lellouche et Philippe Tesson en (12), en (15), sortant de la représentation du discours autre, il formule en le prenant en charge l'appel à la désolidarisation, dont la force injonctive est atténuée par la subordonnée conditionnelle. 


\section{Sous-énonciation et demande de validation}

Les cas de sous-énonciation ont comme point commun d'être des segments où les modérateurs (qui sont ici des modératrices ${ }^{16}$ ) représentent à un invité $\mathrm{X}$ les dires qu'il vient lui-même de tenir. Ainsi, les modératrices opèrent, conformément à leur rôle de médiatrices entre débattants et public (il s'agit ici de représenter les points de vue exprimés sous forme condensée et plus claire, immédiatement rattachée au thème surplombant de l'émission), une reformulation synthétisant le tour de parole précédent et invitent les débattants à valider ou non le discours représenté. Ce lien entre sousénonciation dans la représentation par les modératrices de discours tenus in praesentia et, du point de vue pragmatique, demande de validation, est notable de façon systématique.

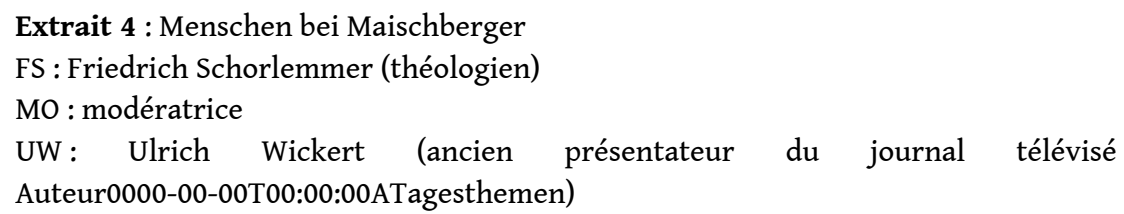

17 Précédemment, la modératrice a invité Tom Buhrow - journaliste qui après des années aux États-Unis revient en Allemagne pour prendre en charge l'animation du journal télévisé du soir le plus populaire, les Tagesthemen - à parler de l'influence de la religion sur les affaires publiques Outre-Atlantique. Ulrich Wickert intervient pour parler du même topic et distingue le rapport au religieux prôné par Jimmy Carter et George W. Bush (en (1)), Friedrich Schorlemmer prend la parole (en (2) et (4)) et critique le discours discours de Bush qui peut pousser selon lui les musulmans au terrorisme. En (7), la modératrice reprend le discours tenu par Schorlemmer (la reformulation semble être amorcée en (5)) : le connecteur «also » (« donc») pose l'énoncé comme conclusion de l'énoncé précédent. Le segment reformulant, sous forme de discours direct attribué à Schorlemmer, est situé en fin de tour («das was wir jetzt gerade sehen (..) ist sozusagen also auch deshalb mit (...) am Hochschaukeln (.) weil es diesen amerikanischen Präsidenten gibt (...) und seine Politik (0.9) seinen Krieg gegen den Irak »/ « ce à quoi nous assistons aujourd'hui (..) est donc pour ainsi dire en train de dégénérer aussi à cause de cela (.) à cause de ce Président américain (...) et sa politique (0.9) sa guerre contre l'Irak»). La modératrice modifie le point de vue de Schorlemmer en liant explicitement l'argument à la question principale de l'émission (« l'affaire des caricatures ») et en établissant prudemment une causalité : la particule de focalisation «auch » («aussi ») porte sur le pronom cataphorique « deshalb ( (« à cause de cela ») et ouvre la voie à une interprétation polyfactorielle. Cette prudence est par ailleurs exprimée par d'autres moyens à valeur métadiscursive qui soulignent l'écart entre reformulé et reformulant: (i) la particule "sozusagen» ("pour ainsi dire»), dans le discours représenté, qui nous semble être attribuable à la locutrice/énonciatrice 1 et montre la prudence de la reformulation proposée, (ii) le groupe participial « ketzerisch gesagt» ("pour le dire de façon provocatrice»), qui ouvre le tour et introduit le discours représenté.

La modératrice clarifie la position de Schorlemmer en la mettant en lien avec le sujet de l'émission. Elle sous-énonce ici puisque (i) elle nuance l'adéquation entre discours reformulant et discours reformulé et puisque (ii) ce tour de parole constitue pragmatiquement une demande de validation (qui est d'ailleurs interprétée comme telle par Schorlemmer en (8)). Ce faisant, la modératrice se conforme à son rôle 
interactionnel consistant à orchestrer les débats en permettant l'expression des points de vue antagonistes: elle permet une prise de position du débattant face aux dires qu'elle lui attribue et se construit un éthos positif.

On trouve un fonctionnement similaire dans l'extrait suivant :

Extrait 5 : Pièces à conviction

MEO : Mohammed El Oifi (Maître de conférences à l'IEP de Paris, spécialiste des médias arabes)

MO : Auteur0000-00-00T00:00:00Amodératrice

Le premier tour de l'extrait constitue la première question adressée à Mohammed El Oifi par la modératrice dans le débat: elle interroge le «choc» de l'invité face aux dessins, lui proposant d'argumenter l'émotion et mettant en jeu deux ethè (« vous êtes d'origine marocaine ", « vous êtes un expert des médias arabes »). En (2), l'invité met à distance l'émotion qui lui est proposée et refonde son éthos sur le logos, se positionnant clairement du côté de l'expertise. Alors que Mohammed El Oifi met en avant la disproportion entre la portée initiale (locale) et la portée finale (internationale) de l'«affaire » et qu'il en impute la responsabilité à un groupe d'individus dans lequel il s'inclut (« les hommes les hommes politiques mais aussi les journalistes nous tous »), le segment que la modératrice lui attribue en (3), sous couvert de synthétiser, attribue la responsabilité de la crise à un seul acteur. Comme ce que nous avons observé dans l'extrait précédent, le tour de parole met en scène un point de vue que la modératrice soumet à la validation de l'invité : la reformulation se présente comme résultat d'un acte de clarification «ce que vous nous dites quand même là » et même de mise au jour de l'intention discursive de l'invité, dont la modératrice se fait l'interprète («si je décrypte bien hein ce que vous voulez nous dire »). Malgré la modification (qui n'est pas insignifiante !) du point de vue représenté, la modératrice se situe ici dans la sousénonciation, comme l'indiquent (i) la subordonnée conditionnelle en «si » et (ii) la locution adverbiale "quand même » que nous comprenons ici, à la suite de Beeching comme un "atténuateur " visant à affaiblir la force assertive de ce qui suit (Beeching 2005: 158). Ces éléments mettent en avant ici la distance entre reformulé et reformulant, en soumettant le discours représenté à l'invité pour validation ou nonvalidation.

\section{Conclusion}

21 Notre étude, examinant les segments où les modérateurs représentent les discours des invités tenus in praesentia, a permis de mettre au jour des constantes dans l'articulation entre postures énonciatives et rôles interactionnels incombant aux modérateurs : la sur-énonciation est liée à des reformulations polémisantes des dires des invités, alors que la sous-énonciation est articulée avec des demandes de validation. Les formes d'expression d'accord avec les points de vue des invités sont, compte tenu de l'éthos de neutralité des modérateurs et de l'effacement énonciatif marquant leurs discours, très minoritaires et apparaissent à travers des postures autres que la co-énonciation. L'originalité de ce travail réside moins dans la mise en avant de la non-transparence du rôle des modérateurs dans les débats télévisés, qui - largement décrite ailleurs constituait notre point de départ, que dans la mise en évidence des fonctionnements énonciatifs précis de leur implication dans le débat, illustrant la pertinence de la prise 
en compte des postures énonciatives pour une analyse critique des discours médiatiques (Rabatel 2017).

\section{BIBLIOGRAPHIE}

Amossy R. (2010). La présentation de soi. Éthos et identité verbale. Paris : PUF.

Amossy R. et Koren R. (éd.) 2004a. « Argumentation et prise de position: pratiques discursives ». Semen 17. Disponible en ligne $:$ https://journals.openedition.org/semen/557 (date de dernière consultation : 10.01.2019)

Amossy R. et Koren R. 2004b. «Introduction ». Semen 17 : 9-18. Disponible en ligne : https:// journals.openedition.org/semen/2305 (date de dernière consultation : 10.01.2019)

Amossy R. et Burger M. 2011. «Introduction : la polémique médiatisée ». Semen 11 : 7-24.

Alexis L. 2016. «Dispositifs télévisuels et mises en scène du désaccord : les cas d'On n'est pas couché et de Ce soir (ou jamais!) ». Cahiers de praxématique 67. Disponible en ligne : http:// journals.openedition.org/praxematique/4473 (date de dernière consultation : 27.01.2019). Beeching K. 2005. «Politeness-induced semantic change: The case of quand même ». Language Variation and Change $17: 155-180$.

Bres J. 2017. « Dialogisme, éléments pour l'analyse ». Recherches en didactique des langues et des cultures 14-2. Disponible en ligne : https://journals.openedition.org/rdlc/1842\#tocto1n3 (date de dernière consultation : 27.01.2019).

Charaudeau P. et Maingueneau D. (dir.) 2002. Dictionnaire d'analyse du discours. Paris : Seuil. Clayman S. et Heritage J. 2002. The News Interview. Journalists and Public Figures. Cambridge : Cambridge University Press.

Doury M. 1995. « Duel sur la cinq : dilogue ou trilogue?», in C. Kerbrat-Orecchioni et C. Plantin (éd.). Le trilogue. Lyon : PUL, 224- 249.

Florea M.-L. 2015. Les nécrologies dans la presse française contemporaine. Une analyse de discours. Thèse de doctorat : Université de Lyon.

Hekmat I. 2012. Des « caricatures de Mahomet » à la construction stéréotypique des identités. Une analyse de discours de débats télévisés allemands et français. Thèse de doctorat : Université de Lyon.

Hekmat I. 2013. « Argumentations de la colère face aux "caricatures de Mahomet" et constructions identitaires ». Le discours et la langue 4-1 : 19-45.

Kerbrat-Orecchioni C. 1980. « La polémique et ses définitions ». in C. Kerbrat-Orecchioni (éd.), Le discours polémique. Lyon : PUL, 3-40.

Kerbrat-Orecchioni C. 1992. Les interactions verbales, tome 2. Paris : Armand Colin.

Kerbrat-Orecchioni C. 2005. Le discours en interaction. Paris : Armand Colin.

Kerbrat-Orecchioni C. 2017. Les débats de l'entre-deux-tours des élections présidentielles françaises.

Constantes et évolutions d'un genre. Paris : L'Harmattan. 
Koren R. 2004. " Argumentation, enjeux et pratique de l'“engagement neutre" : le cas de l'écriture de presse ». Semen $17: 19-40$. Disponible en ligne : https://journals.openedition.org/ semen/2308 (date de dernière consultation : 10.01.2019)

Koren R. 2019. Rhétorique et éthique. Du jugement de valeur. Paris : Classiques Garnier.

Maingueneau D. 1993. Le contexte de l'œuvre littéraire. Énonciation, écrivain, société. Paris : Dunod.

Oprea A. 2012. Le système de la politesse confronté aux défis du talk-show. Thèse de doctorat : Université de Cluj-Napoca.

Pérennec M.-H. 2001. « Le rôle du modérateur dans les débats télévisés », in I. Behr (éd.), Télévision et internet : le parlé et l'écrit. Paris : PIA, Institut d'Allemand de la Sorbonne NouvelleParis III, 13-28.

Prak-Derrington E. 2017. « "Je suis Charlie”. Analyse énonciative et pragmatique d'un slogan de crise ». Cahiers d'études germaniques $73: 19-38$.

Rabatel A. (éd.) 2004a. « Effacement énonciatif et discours rapportés ». Langages 156. Disponible en ligne : https://www.persee.fr/issue/lgge_0458-726x_2004_num_38_156 (date de dernière consultation : 10.01.2019)

Rabatel A. 2004b. «L'effacement énonciatif dans les discours rapportés et ses effets pragmatiques ». Langages $156: 3-17$.

Rabatel A. 2006. «Du rôle des postures de surénonciation et sousénonciation dans les analyses de corpus : l'exemple des reformulations, des connecteurs et des particules discursives ", in M.-C. Guernier, V. Durand-Guerrier et J.-P. Sautot (éd.), Interactions verbales, didactiques et apprentissages. Besançon : PUFC, 221-248.

Rabatel A. 2015. « Postures énonciatives, variable générique et stratégies de positionnement », in J. Angermüller et G. Philippe (éd.), Analyse du discours et dispositifs d'énonciation. Autour des travaux de Dominique Maingueneau. Limoges : Lambert-Lucas, 125-135.

Rabatel A. 2017. Pour une lecture linguistique et critique des médias. Empathie, éthique, point(s) de vue. Limoges : Lambert Lucas.

Rabatel A. et Lepoire S. 2005. « Le dialogisme des discours représentés et des points de vue dans les explications, entre concordance et discordance ». Cahiers de praxématique 45 : 51-76.

Rabatel A. et Chauvin-Vileno A. 2006. « La question de la responsabilité dans l'écriture de presse ». Semen 22, https://journals.openedition.org/semen/2792\#quotation (date de dernière consultation : 28.01.2019)

Rabatel A. et Monte M. 2017. « Pour une analyse énonciative et responsable des discours médiatiques » (entretien avec Alain Rabatel réalisé par Michèle Monte). Mots. Les langages du politique 113 : 117-132.

Sandré M. 2012. « Discours rapporté et stratégies argumentatives : Royal et Sarkozy lors du débat de l'entre-deux tours », Langage \& société 140 : 71-87.

Steuckardt A. 2007. « Usages polémiques de la reformulation », Recherches linguistiques 29 : 55-74.

Vion R. 2001. " "Effacement énonciatif” et stratégies discursives ", in M. De Mattia et A. Joly (éd.), De la syntaxe à la narratologie énonciative. Paris : Ophrys, 331-354.

Yanoshevsky G. (éd.) 2018. Éthique du discours et responsabilité. En hommage à Roselyne Koren. Limoges : Lambert Lucas. 


\section{ANNEXES}

\section{Conventions de transcription}

Nous avons transcrit les débats du corpus en nous appuyant sur les conventions de transcription ICOR ${ }^{17}$, élaborées par des équipes du laboratoire ICAR. Voici les principaux phénomènes transcrits et les conventions associées :

\begin{tabular}{|c|c|}
\hline $\begin{array}{l}\text { Identification } \quad \mathrm{du} \\
\text { locuteur : }\end{array}$ & $\begin{array}{l}\text { Les locuteurs sont identifiés grâce à deux ou trois lettres explicitées } \\
\text { au début de chaque transcription }\end{array}$ \\
\hline Participant incertain : & Insertion d'un point d'interrogation en début de tour. \\
\hline Chevauchements : & $\begin{array}{l}\text { Indiqués par l'insertion d'un « [ " au début des segments chevauchés, } \\
\text { qui sont alignés. La fin du chevauchement n'est pas indiquée. }\end{array}$ \\
\hline Pauses: & $\begin{array}{l}\text { Les pauses courtes (inférieures ou égales à } 0.2 \text { secondes) sont notées : } \\
(.) \\
\text { les pauses moyennes (comprises entre } 0.2 \text { et } 0.4 \text { secondes) : (..) } \\
\text { les pauses longues (comprises entre } 0.4 \text { et } 0.8 \text { secondes) : (...) } \\
\text { La durée des pauses supérieures à } 0.8 \text { secondes est indiquée entre } \\
\text { parenthèses. }\end{array}$ \\
\hline Aspirations : & Notées à l'aide de «.h»" \\
\hline $\begin{array}{l}\text { Montées et chutes } \\
\text { intonatives: }\end{array}$ & $\begin{array}{l}\text { Les montées intonatives sont marquées à l'aide d'un «/", les chutes } \\
\text { intonatives à l'aide de « \» }\end{array}$ \\
\hline $\begin{array}{l}\text { Insistances } \\
\text { prosodiques : }\end{array}$ & Marquées par des majuscules \\
\hline Syllabes inaudibles: & $\begin{array}{l}\text { Indiquées par « } \mathrm{x} » \text {. Le nombre de } \mathrm{x} \text { indique le nombre de syllabes } \\
\text { inaudibles. }\end{array}$ \\
\hline Segments tronqués : & Marqués par un tiret \\
\hline
\end{tabular}

\section{NOTES}

1. La confrontation, constitutive de ce genre de discours, n'est pas uniquement assurée par la parole, elle est portée aussi par les dispositifs des émissions, comme le montre Alexis 2016 qui s'intéresse à deux émissions de débat qui, davantage encore que le dissensus, visent à construire des « clashs".

2. Nous remercions les relecteurs et relectrices de cet article pour leurs observations précieuses.

3. Nous préférons le terme de « discours représenté », circulant dans de nombreuses études sur le dialogisme, à celui de " discours rapporté ", parce que le discours faisant état d'un discours autre (réel ou fictif) est moins rapporté que représenté par le locuteur en fonction de ses motivations (voir Rabatel et Lepoire 2005).

4. Pour une analyse générique des talk-shows, que nous distinguons des débats télévisés envisagés ici, nous renvoyons à Oprea 2012.

5. Douze dessins qui furent appelés «caricatures de Mahomet » publiés dans le journal danois Jyllands Posten fin 2005 avaient déclenché, dans de nombreux pays, protestations, menaces, manifestations - parfois violentes - et débats (voir Hekmat 2012). Les dessins furent publiés en France notamment par Charlie Hebdo, ce qui fit du journal et de ses membres une cible pour divers groupes islamistes. En janvier 2015, deux terroristes se réclamant d'Al Qaida, après avoir 
assassiné un gardien de la paix, pénètrent dans les locaux de Charlie Hebdo et abattent 12 personnes.

6. La construction éthotique dont nous parlons ici relève de l'éthos " montré » et non " dit " (Maingueneau 1993, Amossy 2010). Nous ne pouvons pas approfondir davantage ici la question centrale des liens entre postures énonciatives et éthos, qui est traitée dans l'article de Druetta et Païssa dans ce volume.

7. On observe que l'éthos nourri par les modérateurs n'est pas uniquement un éthos de neutralité : un certain éthos de provocation (s'il est présenté comme feint) est constitutif également de l'image du «bon modérateur ». C'est entre les deux que les modérateurs doivent donc se mouvoir pour apparaître comme n'étant ni de parti pris, ni complaisants.

8. Nous entendons ici, suivant Rabatel, le locuteur comme « instance de production phonique ou graphique de l'énoncé » $(2015: 126)$ et l'énonciateur comme source du point de vue. Dans les cas de dialogisme que nous étudions, les énonciateurs seconds sont liés à des locuteurs.

9. Les conventions de transcription sont explicitées à la fin de l'article. Les traductions françaises du corpus allemand sont de nous. Pour une meilleure compréhension des analyses, nous avons dû parfois préférer la similitude entre texte source et texte cible à l'élégance de la traduction. Les segments soulignés sont de nous également: ils permettent de mettre en valeur les passages constituant le discours représenté par les modérateurs ; les segments en gras sont des segments que nous mettons en avant parce qu'ils sont pointés dans l'analyse.

10. Il est utile d'expliquer en quelques mots qui sont les débattants dont il est question, mais la tâche est plus complexe qu'il n'y paraît puisque leur identité sociale (que nous serions bien en peine de définir précisément) n'est pas nécessairement la même que leur identité discursive pertinente en contexte, identité qui peut leur être assignée ou qu'ils peuvent construire euxmêmes et qui est souvent retravaillée au cours des interactions. Nous choisissons ici d'indiquer à chaque fois les données mentionnées par l'instance d'émission lors de l'ouverture du débat.

11. Nous renvoyons à la définition des "séquences conversationnelles » donnée par Traverso dans Charaudeau et Maingueneau 2002 : 528-530.

12. Pour une analyse de l'argumentation de l'émotion dans ce corpus en lien avec la construction d'identités collectives et individuelles, nous renvoyons à ce que nous avons écrit ailleurs: Hekmat 2013.

13. La représentation polémique du discours autre consiste ici en une représentation qui rend polémique le point de vue présenté et attribué à l'autre. Cette définition est donc à distinguer de celle employée par Steuckardt 2007 qui envisage les reformulations polémiques comme des reformulations anti-orientées du point de vue argumentatif.

14. Nous renvoyons à l'article de Susini dans ce volume qui met au jour, à partir de l'analyse de textes de Pascal, des phénomènes de recouvrement d'une posture énonciative par une autre.

15. Pour une analyse du slogan «Je suis Charlie », nous renvoyons à Prak-Derrington 2017.

16. Nous n'entendons pas genrer de façon systématique cette posture énonciative mais remarquons que, dans ce corpus, le phénomène étudié est observable dans le discours des modératrices uniquement.

17. Disponibles en ligne: http://icar.cnrs.fr/projets/corinte/bandeau_droit/ convention_icor.htm (date de dernière consultation : 27.01.2019). 


\section{RÉSUMÉS}

Dans cet article, ancré dans le champ de l'analyse de discours, on interroge, à partir d'un corpus de débats télévisés, la représentation par les modérateurs des discours tenus par les invités au sein de l'interaction à l'aide de la notion de posture énonciative (notamment Rabatel 2004b et 2017). L'enjeu de cette contribution est ainsi d'analyser les façons dont les modérateurs se situent dans les débats par rapport aux discours des invités : bien qu'ils paraissent être des hôtes neutres et effacés dans l'interaction (notamment en raison de stratégies d'effacement énonciatif : Vion 2001, Rabatel 2004b), ils contribuent fortement à la structuration interactionnelle et argumentative des débats. Des constantes sont mises en évidence : les modérateurs sur-énoncent lorsqu'ils représentent les discours des interactants afin de rendre les échanges plus polémiques et sous-énoncent quand ils demandent aux invités de valider la synthèse proposée. Pour préserver leur ethos de neutralité, les modérateurs évitent la co-énonciation et le cas minoritaire d'expression d'accord avec le point de vue exprimé par les invités se fait à travers une stratégie de sur-énonciation.

Drawing on concepts from French discourse analysis, this article aims to examine the different enunciative postures (Rabatel 2004b, 2017) adopted by moderators in relation to the discourse of their guests, with reference to a corpus of televised political debates. Moderators are often considered to be neutral intermediaries between their guests and audience due to the various forms of enunciative effacement (Vion 2001, Rabatel 2004b) that characterize their speech. However, this contribution seeks to demonstrate the structuring role that moderators play in both enunciative and argumentative terms. Two clear patterns emerge from this analysis: moderators act as over-enunciators when reformulating their guests' speech with a view to making the exchange more polemic and as under-enunciators when asking guests to confirm their syntheses. In the interests of their ethos of neutrality, moderators avoid expressing agreement with the points of view articulated by their invitees, in other words they don't take the position of co-enunciators. As will be demonstrated, a strategy of over-enunciation explains the rare cases of manifest agreement observed.

\section{INDEX}

Mots-clés : Débat télévisé, Discours représenté, Modérateurs, Effacement énonciatif, Postures énonciatives, Sur-énonciation, Sous-énonciation

Keywords : TV debate, Represented speech, Debate moderators, Enunciative effacement, Enunciative postures, Over-enunciation, Under-enunciation

\section{AUTEUR}

\section{IDA HEKMAT}

Université Bourgogne-Franche-Comté, CRIT 